\title{
Entropy and semi-conjugacy in dimension two
}

\author{
MICHAEL HANDEL \\ Department of Mathematics and Computer Science, Lehman College, \\ Bronx, New York 10468, USA
}

(Received 18 May 1987, revised 3 December 1987)

\begin{abstract}
We prove that if a diffeomorphism $f$ of a closed surface is homotopic to and has the same topological entropy as a pseudo-Anosov homeomorphism $g$, then $f$ is semi-conjugate to $g$. As part of the proof, a necessary and sufficient condition is given for a pseudo-orbit of a pseudo-Anosov homeomorphism $g$ to be shadowed by an actual orbit of $g$.
\end{abstract}

\section{0 . Introduction}

We showed in [H] that if $f: M^{2} \rightarrow M^{2}$ is a pseudo-Anosov diffeomorphism and if $g: M \rightarrow M$ is homotopic to $f$, then there is a closed subset $Y \subset M$ such that $g \mid Y$ is semi-conjugate to $f$; i.e. there is a map $\pi: Y \rightarrow M$ such that $\pi \circ g \mid Y=f \circ \pi$. In this paper we give a sufficient condition for $Y$ to be all of $M$ and hence for $g$ to be semi-conjugate to $f$. In general the pre-images of points under the semi-conjugacy will be disconnected.

THEOREM 0.1. Let $f: M^{2} \rightarrow M^{2}$ be a pseudo-Anosov diffeomorphism of a closed surface and let $\mathrm{g}: \mathrm{M}^{2} \rightarrow M^{2}$ be a homeomorphism that is homotopic to $f$. If $\mathrm{g}$ has the same topological entropy as $f$ (it is necessarily at least as big) then $g$ is semi-conjugate to $f$.

The introduction of topological entropy in this context is due to John Smillie who showed that if $g$ has periodic points that are not Nielsen equivalent to periodic points of $f$ (or equivalently if $Y$ does not contain all the periodic points of $g$ ) then the topological entropy of $g$ is strictly bigger than that of $f$.

Let $N_{n}(g)$ be the number of distinct Nielsen classes represented by the fixed point set Fix $\left(g^{n}\right)$ of $g^{n}$. As a corollary of Theorem 0.1, we show (Corollary 3.6) that if the exponential growth rate of $N_{n}(g)$ equals that of $N_{n}(f)$, then $g$ is semi-conjugate to $f$.

In the final section of the paper, we consider extensions of Theorem 0.1 to the more general setting used by Franks for Anosov diffeomorphisms and Fathi for pseudo-Anosov homeomorphisms.

I would like to thank Albert Fathi for his helpful comments and careful reading of an earlier manuscript for this paper. 


\section{Notation and definitions}

Throughout this paper $M$ is a closed surface, $f$ is a pseudo-Anosov diffeomorphism of $M$ and $g$ is a homeomorphism of $M$ that is homotopic to $f$. Following A. B. Katok, we say that the $f$-orbit of $x$ is globally shadowed by the $g$-orbit of $y$ if there are lifts $\tilde{f}, \tilde{g}, \tilde{x}$ and $\tilde{y}$ to the universal cover $\tilde{M}$ of $M$ such that $\tilde{D}\left(\tilde{f}^{n}(\tilde{x}), \tilde{g}^{n}(\tilde{y})\right)<K$ for all $n$ and some fixed $K$ where $\tilde{D}$ is an equivariant metric on $\tilde{M}$. Define $Y=\{y \in M$ : the $g$-orbit of $y$ is globally shadowed by the $f$-orbit of some $x \in M\}$ and let $\pi: Y \rightarrow M$ be the map which takes $y$ to its associated $x$. We showed in [H] that $Y$ is a closed subset of $M$, that $\pi$ is a surjective map and that $\pi$ semi-conjugates $g \mid Y$ onto $f$.

Our proof takes place in the universal cover $\tilde{M}$ of $M$ and uses the invariant stable and unstable foliations (with singularities) on $\tilde{M}$ that are lifted from the invariant stable and unstable foliations (with singularities) that characterize $f$. Readers that are not familiar with pseudo-Anosov diffeomorphisms should consult [T], [F-L-P] or $[\mathbf{H}-\mathbf{T}]$ as required. For any point $\tilde{p} \in \tilde{M}$, we denote the unstable leaf containing $\tilde{p}$ by $\tilde{\mu}(\tilde{p})$ and the stable leaf containing $\tilde{p}$ by $\tilde{\sigma}(\tilde{p})$. When the unstable leaf through $\tilde{p}$ contains a singularity we assume that $\tilde{\mu}(\tilde{p})$ contains that singularity and each of the finitely many leaves emanating from that singularity; similarly for $\tilde{\sigma}(\tilde{p})$. The transverse measures for the foliations determine equivariant pseudo-metrics $\tilde{D}_{s}: \tilde{M} \times \tilde{M} \rightarrow[0, \infty)$ and $\tilde{D}_{u}: \tilde{M} \times \tilde{M} \rightarrow[0, \infty)$ satisfying $\tilde{D}_{u}\left(\tilde{f} \tilde{x}_{1}, \tilde{f} \tilde{x}_{2}\right)=\lambda \tilde{D}_{u}\left(\tilde{x}_{1}, \tilde{x}_{2}\right)$ and $\tilde{D}_{s}\left(\tilde{f}^{-1} \tilde{x}_{1}, \tilde{f}^{-1} \tilde{x}_{2}\right)=\lambda \tilde{D}_{s}\left(\tilde{x}_{1}, \tilde{x}_{2}\right)$ for all $\tilde{x}_{1}, \tilde{x}_{2} \in \tilde{M}$ and all lifts $\tilde{f}$ of $f$ where $\lambda>1$ is the expansion constant for $f$. We will use the equivariant metric $\tilde{D}$ on $\tilde{M}$ defined by $\tilde{D}=\tilde{D}_{u}+\tilde{D}_{s}$.

Since $Y$ is unchanged when $f$ and $g$ are replaced by $f^{k}$ and $g^{k}$ for any $k>0$, we may assume that $f$ fixes each singularity of the stable and unstable foliations, that $f$ maps each stable and unstable half-leaf that initiates at a singularity to itself, and that $\lambda>4$.

An efficient path (see figure 1.1) between $\tilde{x}$ and $\tilde{y}$ is a path that is made up of segments of stable and unstable leaves, that has the minimum possible number of such segments and that is shortest in the $\tilde{D}$-metric.

Choose $K>0$ so that for any pair $\tilde{f}$ and $\tilde{g}$ of equivariantly homotopic lifts of $f$ and $g$, and for all $\tilde{x} \in \tilde{M}, \tilde{D}(\tilde{f}(\tilde{x}), \tilde{g}(\tilde{x}))<K$. It follows that if $\tilde{D}_{u}\left(\tilde{x}_{1}, \tilde{x}_{2}\right)>K$ then

$$
\tilde{D}_{u}\left(\tilde{g}\left(\tilde{x}_{1}\right), \tilde{g}\left(\tilde{x}_{2}\right)\right)>\tilde{D}_{u}\left(\tilde{x}_{1}, \tilde{x}_{2}\right)+K
$$

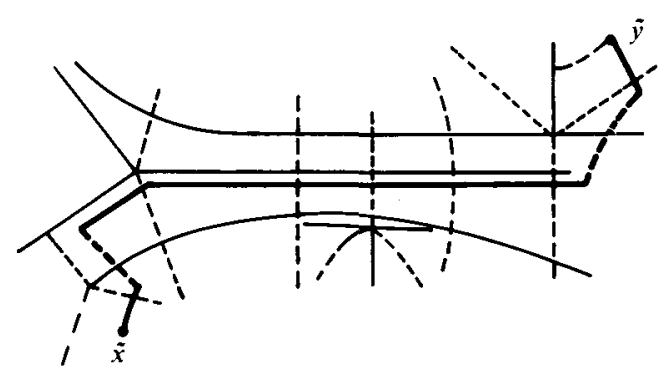

Figure 1.1 
and

$$
\tilde{D}_{u}\left(\tilde{f}\left(\tilde{x}_{1}\right), \tilde{g}\left(\tilde{x}_{2}\right)\right)>\tilde{D}_{u}\left(\tilde{x}_{1}, \tilde{x}_{2}\right)+2 K
$$

similarly if $\tilde{D}_{s}\left(\tilde{x}_{1}, \tilde{x}_{2}\right)>K$ then

$$
\tilde{D}_{s}\left(\tilde{g}^{-1}\left(\tilde{x}_{1}\right), \tilde{g}^{-1}\left(\tilde{x}_{2}\right)\right)>\tilde{D}_{s}\left(\tilde{x}_{1}, \tilde{x}_{2}\right)+K
$$

and

$$
\tilde{D}_{s}\left(\tilde{f}^{-1}\left(\tilde{x}_{1}\right), \tilde{g}^{-1}\left(\tilde{x}_{2}\right)\right)>\tilde{D}_{s}\left(\tilde{x}_{1}, \tilde{x}_{2}\right)+2 K .
$$

If $\tilde{z}$ is a singularity of the foliations and if $W^{u}$ is a component of $\tilde{M}-\tilde{\sigma}(\tilde{z})$ then $W^{u}$ is called an unstable wedge based at $\tilde{z}$. See figure 1.2. For each unstable wedge $W^{u}$, we define $E^{u}$, an unstable envelope based at $\tilde{z}$ to be the component of $\left\{\tilde{x} \in W^{u}: \tilde{D}_{u}(\tilde{x}, \tilde{\sigma}(\tilde{z}))>K\right\}$ that intersects the unstable half-leaf in $W^{u}$ originating at $\tilde{z}$. Note that if $\tilde{g}$ is the lift of $g$ that is equivariantly homotopic to the lift $\tilde{f}$ of $f$ that fixes $\tilde{z}$, and if $E^{u}$ is an unstable envelope based at $\tilde{z}$, then $\tilde{g}\left(E^{u}\right) \subset E^{u}$. Stable wedges and envelopes are defined similarly.

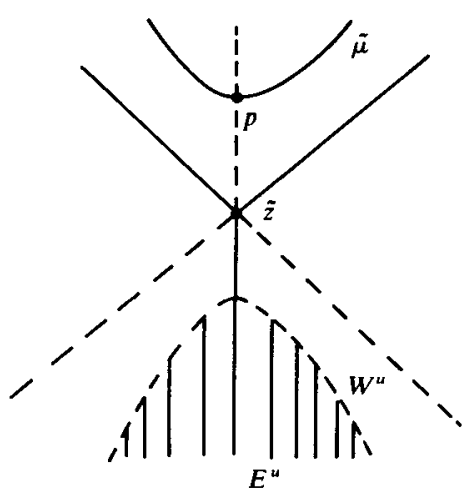

FIGURE 1.2

Remark 1.1. If $\tilde{x} \in \tilde{M}$ and $\tilde{\sigma}$ is a stable leaf such that $\tilde{D}_{u}(\tilde{x}, \tilde{\sigma})>K$, then for all $n \geq 0, \tilde{g}^{n}(\tilde{x})$ is in the component of $\tilde{M}-\tilde{f}^{n}(\tilde{\sigma})$ that is the image under $\tilde{f}^{n}$ of the component of $\tilde{M}-\tilde{\sigma}$ that contains $\tilde{x}$. If $\tilde{\sigma} \subset W^{u}$ intersects the unstable half-leaf originating at $\tilde{z}$, then $\tilde{f}^{n}(\tilde{\sigma}) \subset E^{u}$ for all sufficiently large $n$. It follows that if $\tilde{x} \in\left\{W^{u}: \tilde{D}_{u}(\tilde{x}, \tilde{\sigma}(\tilde{z}))>K\right\}$, then $\tilde{g}^{n}(\tilde{x}) \in E^{u}$ for all sufficiently large $n$.

The stable variation along a path $\rho$ is the maximum $\tilde{D}_{s}$-distance between two points on $\rho$. Note that if $\rho$ has a stable variation less than $K$, then so does $\tilde{g}(\rho)$.

\section{An equivalent condition for semi-conjugacy}

The construction in [H] shows that the homeomorphism $g$ is semi-conjugate to $f$ if for each $y \in M$, there exists $x \in M$ such that the $g$-orbit of $y$ is globally shadowed by the $f$-orbit of $x$. For Anosov diffeomorphisms, $x$ can always be found by the standard shadowing techniques. In the pseudo-Anosov category, these techniques break down and the inability to solve the shadowing problem can be detected by 
the asymptotic behavior of the orbit of a lift $\tilde{y}$ of $y$ with respect to some lift $\tilde{g}_{0}$ of $g$ as follows.

Proposition 2.1: If the g-orbit of $y$ is not globally shadowed by some f-orbit, then there is a singularity $\tilde{z}$ and disjoint stable and unstable envelopes $E^{s}$ and $E^{u}$ based at $\tilde{z}$ such that $\tilde{g}^{-n}(\tilde{y}) \in E^{s}$ and $\tilde{g}^{n}(\tilde{y}) \in E^{u}$ for all sufficiently large $n$, where $\tilde{g}$ is the lift of $g$ that is equivariantly homotopic to the lift $\tilde{f}$ of $f$ that fixes $\tilde{z}$.

Proof of Proposition 2.1. Choose lifts $\tilde{y}$ of $y, \tilde{g}_{0}$ of $g$ and $\tilde{f}_{0}$ of $f$ such that $\tilde{g}_{0}$ and $\tilde{f}_{0}$ are equivariantly homotopic. We search for an $\tilde{f}_{0}$-orbit that shadows the $\tilde{g}_{0}$-orbit of $\tilde{y}$; the construction fails only when we encounter the desired lift $\tilde{g}$.

We first find a stable leaf $\tilde{\sigma}$ such that $\tilde{D}_{u}\left(\tilde{g}_{0}^{n}(\tilde{y}), \tilde{f}_{0}^{n}(\tilde{\sigma})\right)<K$ for all $n>0$. If $\tilde{\mu}(\tilde{y})$ is non-singular, choose an orientation for $\tilde{\mu}(\tilde{y})$ and denote this oriented leaf by $\tilde{\mu}_{0}(\tilde{y})$. If $\tilde{\mu}(\tilde{y})$ contains a singularity $\tilde{z}_{0}$, then either $\tilde{g}_{0}^{n}(\tilde{y}) \in N_{K}\left(\tilde{f}_{0}^{n}\left(\tilde{\sigma}\left(\tilde{z}_{0}\right)\right)\right)$ for all $n>0$ and we're done, or (Remark 1.1) $\tilde{g}_{0}^{n}(\tilde{y}) \in \tilde{f}_{0}^{n}\left(E^{u}\right)$ for some unstable envelope $E^{u}$ based at $\bar{z}_{0}$ and for all sufficiently large $n$. In this latter case denote the oriented unstable half-leaf initiating at $\tilde{z}_{0}$ and intersecting $E^{u}$ by $\tilde{\mu}_{0}(\tilde{y})$.

If the stable leaf $\tilde{\sigma}(\tilde{p})$ through $\tilde{p} \in \tilde{\mu}_{0}(\tilde{y})$ is non-singular, then each $\tilde{f}_{0}^{n} \tilde{\sigma}(\tilde{p})$ separates $\tilde{M}$ into two components which we label + and - according to the orientation on $\tilde{f}_{0}^{n}\left(\tilde{\mu}_{0}(\tilde{y})\right)$ induced from that on $\tilde{\mu}_{0}(\tilde{y})$. We define a function $T:\{\tilde{p} \in$ $\tilde{\mu}_{0}(\tilde{y}): \tilde{\sigma}(\tilde{p})$ is non-singular $\} \rightarrow\{+, 0,-\}$ as follows. If $\tilde{D}_{u}\left(\tilde{g}_{0}^{n}(\tilde{y}), \tilde{f}_{0}^{n}(\tilde{\sigma}(\tilde{p}))\right)<K$ for all $n>0$ (i.e. if $\tilde{\sigma}(\tilde{p})$ is the desired stable leaf) then $T(p)=0$. Otherwise, (Remark 1.1), $\tilde{g}_{0}^{n}(\tilde{y})$ is either contained in the + component of the complement of $\tilde{f}_{0}^{n}(\tilde{\sigma}(\tilde{p}))$ for all sufficiently large $n$ or it is contained in the - component for all sufficiently large $n$; in the former case $T(p)=+$ and in the latter case $T(p)=-$. Note that $T^{-1}(-)$ and $T^{-1}(+)$ are open subsets of the domain, that the image of $T$ contains both + and - and that if the domain is ordered according to the orientation on $\tilde{\mu}_{0}(\tilde{y})$ and the range is ordered by $+<0<-$, then $T$ is order preserving.

Let $\tilde{q}$ be the intersection of the closures of $T^{-1}(-)$ and $T^{-1}(+)$. If $\tilde{\sigma}(\tilde{q})$ is not the desired stable leaf then $\tilde{\sigma}(\tilde{q})$ contains a singularity $\tilde{z}_{1}$ and (Remark 1.1) there is an unstable wedge $W_{1}^{u}$ based at $\tilde{z}_{1}$ that is disjoint from $\tilde{\mu}(\tilde{y})$ such that $\tilde{g}_{0}^{n}(\tilde{y}) \in$ $\tilde{f}_{0}^{n}\left(E_{1}^{u}\right)$ for all sufficiently large $n$, where $E_{1}^{u}$ is the unstable envelope for $W_{1}^{u}$. See figure 2.1. Let $\tilde{\mu}_{1}$ be the oriented unstable half leaf in $W_{1}^{u}$ whose negative end converges to $\tilde{z}_{1}$. There is a map $T_{1}:\left\{\tilde{p} \in \tilde{\mu}_{1}: \tilde{\sigma}(\tilde{p})\right.$ is non-singular $\} \rightarrow\{+, 0,-\}$ as above. Iterating this argument we either find the desired stable leaf $\tilde{\sigma}$ or we find

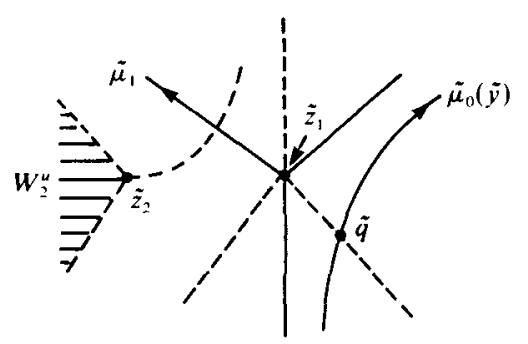

Figure 2.1 
sequences $\left\{\tilde{z}_{i}\right\}$ of singularities, $\left\{E_{i}^{u}\right\}$ of unstable envelopes based at $\tilde{z}_{i}$ and $\left\{W_{i}^{u} \supset E_{i}^{u}\right\}$ of unstable wedges based at $\tilde{z}_{i}$ such that $\tilde{g}_{0}^{n}(\tilde{y}) \in f_{0}^{n}\left(E_{i}^{u}\right)$ for all sufficiently large $n$ and such that $\tilde{z}_{i} \notin W_{j}^{u}$ for $j>i$. In particular, note that $\tilde{D}_{u}\left(\tilde{y}, \tilde{z}_{i}\right)<K$ for all $i$.

Let $W_{i}^{s}$ be the stable wedge based at $\tilde{z}_{i}$ that contains $\tilde{y}$ and let $E_{i}^{s}$ be the stable envelope based at $\tilde{z}_{i}$ that is contained in $W_{i}^{s}$. Then $W_{i}^{s} \cap W_{i}^{u}=\varnothing$ for all $i$. Since $\tilde{D}_{u}\left(\tilde{y}, \tilde{z}_{i}\right)<K$ for all $i$ and since the set of singularities is discrete, there exists $i>0$ such that $\tilde{D}_{s}\left(\tilde{y}, \tilde{z}_{i}\right)>K$. In this case $\tilde{y} \in W_{i}^{s}$ and the conclusions of the proposition are satisfied by $\tilde{z}=\tilde{z}_{i}, E^{s}=E_{i}^{s}$ and $E^{u}=E_{i}^{u}$.

A similar argument produces an unstable leaf $\tilde{\mu}$ such that $\tilde{D}_{s}\left(\tilde{g}_{0}^{n}(\tilde{y}), \tilde{f}_{0}^{n}(\tilde{\mu})\right)<K$ for all $n<0$. If $\tilde{\mu}$ and $\tilde{\sigma}$ intersect then their intersection is a point $\tilde{x}$ whose $\tilde{f}_{0}$-orbit shadows the $\tilde{g}_{0}$-orbit of $\tilde{y}$. If $\tilde{\mu}$ and $\tilde{\sigma}$ are disjoint, choose $p \in \tilde{\mu}$ so that $\tilde{D}_{u}(\tilde{\sigma}(\tilde{p}), \tilde{\sigma}) \leq$ $\tilde{D}_{u}(\tilde{\sigma}(\tilde{q}), \tilde{\sigma})$ for all $\tilde{q} \in \tilde{\mu}$. Then $\tilde{\sigma}(\tilde{p})$ contains a singularity $\tilde{z}$ and $\tilde{\sigma}$ is contained in an unstable wedge $W^{u}$ that is disjoint from $\tilde{\mu}$. Let $W^{s}$ be the stable wedge that contains $\tilde{\mu}$ and let $E^{s}$ and $E^{u}$ be the envelopes determined by $W^{s}$ and $W^{u}$. The conclusions of the proposition are satisfied by $\tilde{z}, E^{s}$ and $E^{u}$.

\section{Computing entropy}

In this section we assume that $g$ is not semi-conjugate to $f$ and show that the entropy of $g$ is strictly greater than that of $f$. We begin with some preliminary definitions and lemmas.

Since $g$ is not semi-conjugate to $f$ there is an orbit of $g$ that is not globally shadowed by an orbit of $f$. Choose $\tilde{\mathrm{g}}, \tilde{f}, \tilde{z}, \tilde{y}, E^{s}$, and $E^{u}$ as in Proposition 2.1; recall that $K>\sup \left\{\tilde{D}_{u}(\tilde{f}(\tilde{x}), \tilde{g}(\tilde{x})): \tilde{x} \in \tilde{M}\right\}$ and that after replacing $g$ by an iterate itself (and hence $\lambda$ by a power of itself) if necessary, we may assume that $\lambda>4$. Let $c_{1}=1 /(\lambda-1)<\frac{1}{3}$ and note that $1+\lambda+\lambda^{2}+\cdots+\lambda^{n-1}<c_{1} \lambda^{n}$ for all $n>0$.

LEMMA 3.1. There exists $0<c_{2}<1$ such that if $\tilde{D}_{u}(\tilde{a}, \tilde{b})>K$ then $\tilde{D}_{u}\left(\tilde{g}^{n}(\tilde{a}), \tilde{g}^{n}(\tilde{b})\right)>$ $c_{2} \lambda^{n} \tilde{D}_{u}(\tilde{a}, \tilde{b})$ for all $\tilde{a}, \tilde{b} \in \tilde{M}$.

Proof of Lemma 3.1. Repeated application of the fact that

$$
\tilde{D}_{u}(\tilde{g}(\tilde{b}), \tilde{g}(\tilde{a}))>\tilde{D}_{u}(\tilde{f}(\tilde{b}), \tilde{f}(\tilde{a}))-2 K=\lambda \tilde{D}_{u}(\tilde{b}, \tilde{a})-2 K
$$

leads to

$$
\tilde{D}_{u}\left(\tilde{g}^{n}(\tilde{b}), \tilde{g}^{n}(\tilde{a})\right) \geq \lambda^{n}\left(\tilde{D}_{u}(\tilde{a}, \tilde{b})-2 K c_{1}\right)
$$

Choose $c_{2}=\frac{1}{3}$.

For any two points $\tilde{a}$ and $\tilde{b}$ in $\tilde{M}$, let $u(\tilde{a}, \tilde{b})$ be the number of unstable segments in an efficient path between $\tilde{a}$ and $\tilde{b}$.

Lemma 3.2. There exists $C>0$ such that $u(\tilde{\mathbf{g}}(\tilde{a}), \tilde{\mathbf{g}}(\tilde{b}))<\boldsymbol{u}(\tilde{a}, \tilde{b})+C$ for all $\tilde{a}, \tilde{b} \in \tilde{M}$. Proof of Lemma 3.2. It is clear from the definition that

$$
u(\tilde{\mathbf{g}}(\tilde{a}), \tilde{\mathbf{g}}(\tilde{b})) \leq u(\tilde{\mathbf{g}}(\tilde{a}), \tilde{f}(\tilde{a}))+u(\tilde{f}(\tilde{a}), \tilde{f}(\tilde{b}))+u(\tilde{f}(\tilde{b}), \tilde{g}(\tilde{b})) \text {. }
$$

The lemma therefore follows from the fact that there is a uniform bound to the distance between $\tilde{f}(\tilde{\boldsymbol{x}})$ and $\tilde{\boldsymbol{g}}(\tilde{\boldsymbol{x}})$ and the fact that $\tilde{f}$ carries efficient paths to efficient paths. 


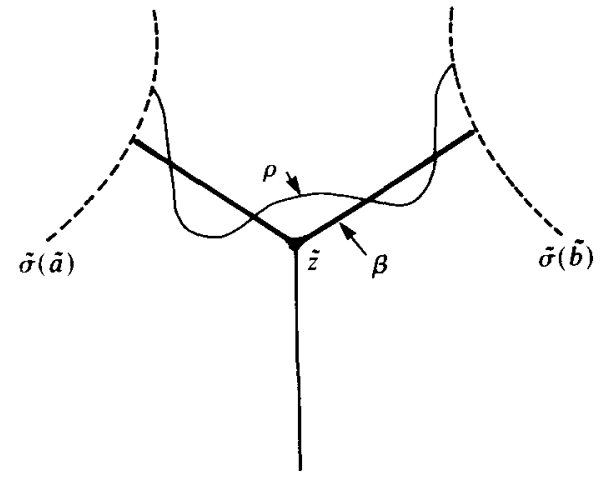

(a)

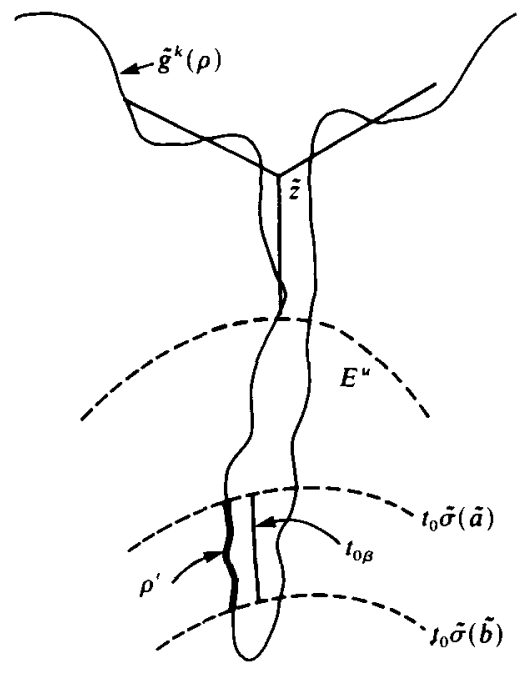

(b)

FIGURE 3.1

LEMMA 3.3. Given $T>K$, let $\beta \subset \tilde{\mu}(\tilde{z})$ be the union of the two T-length initial segments of the unstable half leaves contained in the frontier of the stable wedge containing $E^{s}$ (see figure 3.1). Denote the endpoints of $\beta$ by $\{\tilde{a}, \tilde{b}\}$. Then for all sufficiently large $T$, there exists $k>0$ and a covering translation $t_{0}$ such that if $\rho$ :

is a path connecting $\tilde{\sigma}(\tilde{a})$ to $\tilde{\sigma}(\tilde{b})$ with stable variation less

than $K$, with $\tilde{D}_{s}(\tilde{x}, \beta)<K+1$ for each $\tilde{x} \in \rho$ and with interior disjoint from $\tilde{\sigma}(\tilde{a}) \cup \tilde{\sigma}(\tilde{b})$

then there is a subpath $\rho^{\prime} \subset \tilde{g}^{k}(\rho)$ such that $t_{0}^{-1} \rho^{\prime}$ satisfies (*). Moreover, $\rho^{\prime}$ and the two endpoints of $\tilde{g}^{k}(\rho)$ are contained in three distinct unstable envelopes for $\tilde{z}$.

Proof of Lemma 3.3. For sufficiently large $n, \tilde{g}^{n-1}\left(E^{s}\right) \cap E^{u}$ is non-empty. Thus if $T$ is sufficiently large, $\tilde{g}^{n-1}\left(E^{s}\right) \cup E^{u}$ separates $\tilde{g}^{n}(a)$ from $\tilde{g}^{n}(b)$ and since $\rho \cap \tilde{g}^{-1}\left(E^{s}\right)=\phi, \tilde{g}^{n}(\rho) \cap E^{u}$ is non-empty. A similar argument allows us to choose $n>0$ so that for all sufficiently large $T, \tilde{g}^{n}(\rho) \cap E^{u}$ contains two points $\tilde{p}, \tilde{q} \in E^{u}$ satisfying $\tilde{D}_{u}(\tilde{p}, \tilde{q})>K$.

As $m$ increases $\tilde{D}_{u}\left(\tilde{g}^{m}(\tilde{p}), \tilde{g}^{m}(\tilde{q})\right)$ grows exponentially in $m$ while $u\left(\tilde{g}^{m}(\tilde{p}), \tilde{g}^{m}(\tilde{q})\right)$ grows linearly in $m$. For sufficiently large $m$ an efficient path $\gamma^{\prime}$ between $\tilde{g}^{m}(\tilde{p})$ and $\tilde{g}^{m}(\tilde{q})$ contains arbitrarily long unstable leaf segments and hence unstable leaf segments that are nearly parallel to translates of $\beta$. In particular, there is a covering translation $t_{0}$ and an unstable leaf segment $\beta^{\prime} \subset N_{1}\left(t_{0} \beta\right) \cap \gamma^{\prime}$ connecting $t_{0} \tilde{\sigma}(\tilde{a})$ to $t_{0} \tilde{\sigma}(\tilde{b})$. Since $\gamma^{\prime}$ is efficient, the stable leaves through the endpoints of $\beta^{\prime}$ separate $\tilde{g}^{m}(\tilde{p})$ from $\tilde{g}^{m}(\tilde{q})$ and there is an $\operatorname{arc} \rho^{\prime} \subset \tilde{g}^{m+n}(\rho)$ that connects these stable leaves but is otherwise disjoint from them. Let $k=m+n$. The stable variation of $\rho^{\prime}$ is bounded by the stable variation of $\tilde{g}^{k}(\rho)$ and is therefore less than $K$. Since the unstable leaf that contains $\beta^{\prime}$ separates $\tilde{g}^{m}(\tilde{p})$ from $\tilde{g}^{m}(\tilde{q})$, 
there exists $\tilde{x}_{0} \in \tilde{g}^{k}(\rho)$ with $D_{s}\left(\tilde{x}_{0}, \beta^{\prime}\right)=0$. It follows that $\tilde{D}_{s}\left(\tilde{x}, \beta^{\prime}\right)<K$ and hence that $\tilde{D}_{s}\left(\tilde{x}, t_{0} \beta\right)<K+1$ for all $\tilde{x} \in \rho^{\prime}$.

We now assume that $T$ is chosen as in Lemma 3.3. We say that a path $\rho \subset \tilde{M}$ and a covering translation $t$ are an admissible pair if $t^{-1}(\rho)$ satisfies $(*)$.

We say that a path $\rho$ is admissible if it is part of an admissible pair. Note that if $(\rho, t)$ is an admissible pair, then there is a subpath $\rho^{\prime} \subset \tilde{g}(\rho)$ such that $\left(\rho^{\prime}, \tilde{g} t \tilde{g}^{-1}\right)$ is an admissible pair. If $\alpha$ is an arc containing $\rho$, we say that the admissible pair $(\rho, t)$ is $\alpha$-forced if the stable leaves through the endpoints of $\rho$ each separate the endpoints of $\alpha$; we say that $(\rho, t)$ is $\alpha$-unforced if there is a singularity $\tilde{z}_{i}$ such that $\rho$ and the two endpoints of $\alpha$ are contained in three distinct unstable envelopes for $\tilde{z}_{i}$. Thus Lemma 3.3 states that for any admissible pair ( $\rho$, identity), there is a subpath $\rho^{\prime} \subset \tilde{g}^{k}(\rho)$ such that $\left(\rho^{\prime}, t_{0}\right)$ is a $\tilde{g}^{k}(\rho)$-unforced admissible pair.

For any path $\alpha$, let $F_{n}(\alpha)$ [respectively $U_{n}(\alpha)$ ] be the maximum cardinality of a collection $\{(\rho(i), t(i))\}$ of $\tilde{g}^{n}(\alpha)$-forced [respectively $\tilde{g}^{n}(\alpha)$-unforced] admissible pairs where each $\rho(i) \subset \tilde{g}^{n}(\alpha)$ and the $t(i)$ 's are distinct.

As a corollary to the (second paragraph of the) proof of Lemma 3.3, we have the following estimate on $F_{n}(\rho)$.

COROllary 3.4. There exists $c_{3}>0$ such that $F_{n}(\rho)>c_{3} \lambda^{n}$ for all admissible paths.

After replacing $g$ by an iterate of itself if necessary, we may assume that the constant $k$ of Lemma 3.3 equals 1 , that $c_{3} \lambda>4$ and that $c_{1}+\left(2 / c_{3} \lambda\right)<1$. Choose $1>d>0$ so that $\left[d \lambda^{j}+1\right]<c_{3} \lambda^{j} / 2$ for all $j \geq 1$ where [ ] denotes the greatest integer function. Note that

$$
\sum_{i=1}^{n}\left[d \lambda^{i-1}+1\right] \leq 1+\sum_{i=1}^{n-1}\left(c_{3} \lambda^{i} / 2\right) \leq 1+c_{1} c_{3} \lambda^{n} / 2=\left(c_{1}+2 / c_{3} \lambda^{n}\right)\left(c_{3} \lambda^{n} / 2\right)<c_{3} \lambda^{n} / 2
$$

The following lemma shows that for any admissible path $\rho \subset \tilde{M}$, the exponential growth rate of $U_{n}(\rho)$ is strictly larger than $\log (\lambda)$.

Lemma 3.5. Let $\rho$ be an admissible path. Then $U_{n}(\rho)>B_{n}$ where $\left(B_{n}\right)$ is a sequence satisfying $B_{n+1}>(\lambda+d) B_{n}$.

Proof of Lemma 3.5. If $(\rho(i, j), t(i, j))$ is a $\tilde{g}^{i}(\rho)$-unforced admissible pair for some $i \geq 1$, then there is a unique singularity $\tilde{z}(i, j)$ such that $\tilde{g}^{n-i}(\rho(i, j))$ and the two endpoints of $\tilde{g}^{n}(\rho)$ are contained in three distinct envelopes of $\tilde{f}^{n-i} \tilde{z}(i, j)$ for all $n \geq i \geq 1$. The first step in the proof is an inductive construction $(i \geq 1)$ of a collection $P_{i}(\rho)=\left\{(\rho(i, j), t(i, j)): 1 \leq j \leq\left[d \lambda^{i-1}+1\right]\right\}$ of $\tilde{g}^{i}(\rho)$-unforced admissible pairs such that for each $n \geq 1$, all the elements of $\left\{\tilde{f}^{n-i}(\tilde{z}(i, j)): 1 \leq i \leq n ; 1 \leq j \leq\left[d \lambda^{i-1}+1\right]\right\}$ are distinct. See figure 3.2. In particular, for all $1 \leq i, k \leq n, 1 \leq j \leq\left[d \lambda^{i-1}+1\right]$, and $1 \leq l \leq\left[d \lambda^{k-1}+1\right], \tilde{g}^{n-i}(\rho(i, j))$ and $\tilde{g}^{n-k}(\rho(k, l))$ do not intersect a common stable leaf.

There is no loss in assuming that $\rho$ satisfies $(*)$. Define $P_{1}(\rho)=\{(\rho(1,1), t(1,1))\}$ to be the admissible pair produced by Lemma 3.3 ; note that $\tilde{z}(1,1)=\tilde{z}$. We now assume that $P_{1}(\rho), \ldots, P_{n}(\rho)$ have been defined. Corollary 3.4 states that 

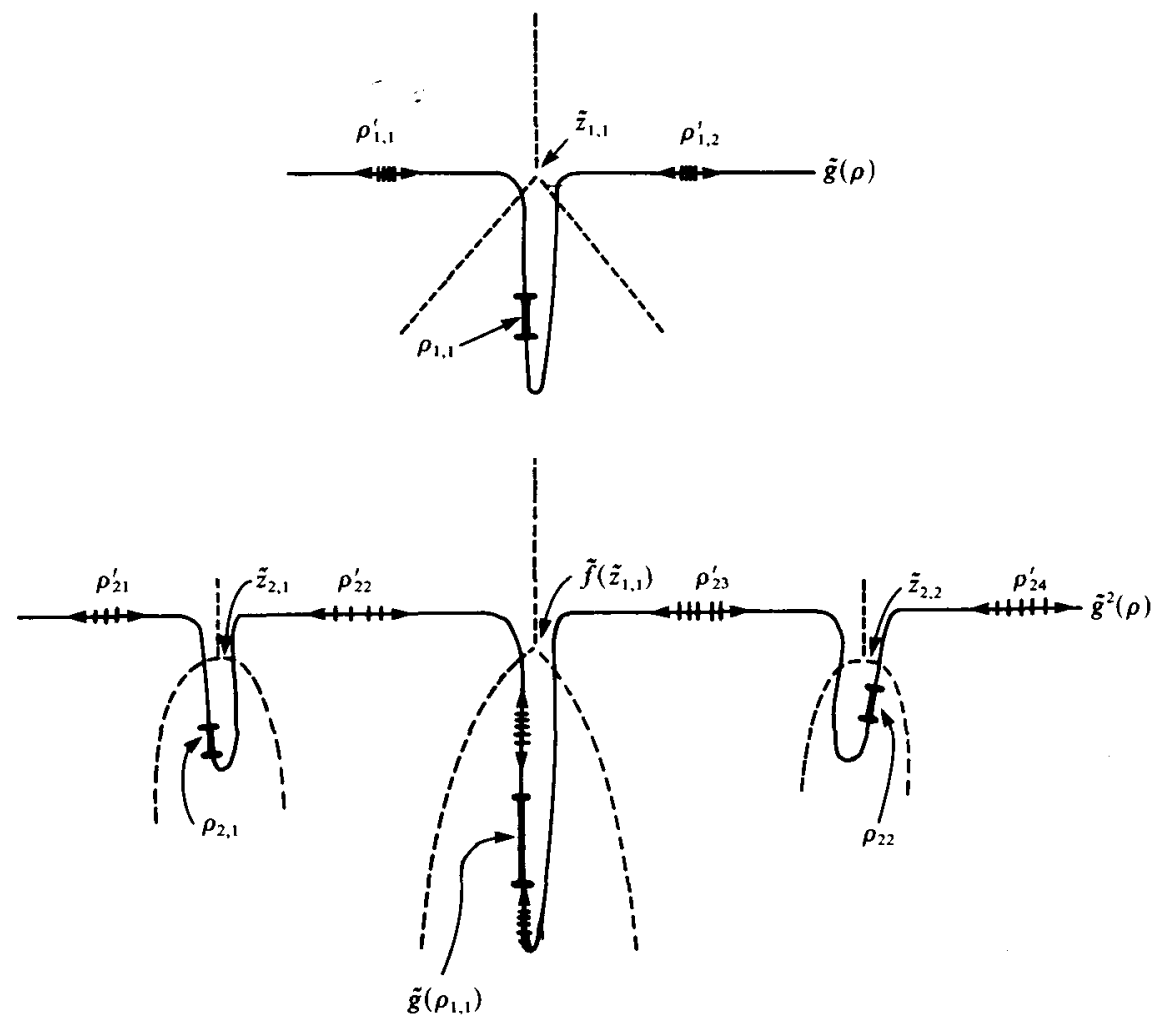

FIGURE 3.2

$F_{n}(\rho)>c_{3} \lambda^{n}$. Since

$$
\sum_{i=1}^{n}\left[d \lambda^{i-1}+1\right]<c_{3} \lambda^{n} / 2
$$

there are $\left[d \lambda^{n}+1\right]<c_{3} \lambda^{n} / 2 \tilde{g}^{n}(\rho)$-forced admissible pairs $\left\{\left(\rho^{\prime}(n, j), t^{\prime}(n, j)\right)\right\}$ such that

$$
\left\{t^{\prime}(n, j) \tilde{z}\right\} \cap\left\{\tilde{f}^{n-i}(\tilde{z}(i, l)): 1 \leq i \leq n ; 1 \leq l \leq\left[d \lambda^{i-1}+1\right]\right\}=\varnothing .
$$

Applying Lemma 3.3 to $t^{\prime}(n, j)^{-1} \rho^{\prime}(n, j)$, we obtain a $\tilde{\mathrm{g}} t^{\prime}(n, j)^{-1} \tilde{\mathrm{g}}^{n}(\rho)$-unforced admissible pair $\left(\alpha(j), t_{0}\right)$. Define

$$
(\rho(n+1, j), t(n+1, j))=\left(t^{\prime \prime}(n, j) \alpha(j), t^{\prime \prime}(n, j) t_{0}\right)
$$

where $t^{\prime \prime}(n, j)$ is the covering translation $\tilde{g} t^{\prime}(n, j) \tilde{g}^{-1}$, and note that $\tilde{z}(n+1, j)=$ $\tilde{f} t^{\prime}(n, j) \tilde{z}$. This completes the construction of $P_{n+1}(\rho)$.

We next define collections $Q_{n}(n \geq 1)$ of $\tilde{g}^{n}(\rho)$-unforced admissible paths by $Q_{0}(\rho)=\rho$ and $(n \geq 1) Q_{n}(\rho)=\bigcup P_{i(k)}\left(P_{i(k-1)}\left(\ldots P_{i(1)}(\rho)\right)\right)$ where the union is taken over all ordered $k$-tuples $(1 \leq k \leq n)$ of positive integers $i(1), \ldots, i(k)$ whose sum equals $n$ and where $P_{i}$ of a union is the union of the $P_{i}$ 's. Thus for each $(\beta, t) \in Q_{n}(\rho)$ there is a sequence of admissible paths

$$
\tau_{i(1)} \in P_{i(1)}(\rho), \tau_{i(2)} \in P_{i(2)}\left(\tau_{i(1)}\right), \ldots, \tau_{i(k)}=\beta \in P_{i(k)}\left(\tau_{i(k-1)}\right) \text {. }
$$


If $\left(\beta^{\prime}, t^{\prime}\right)$ is another element of $Q_{n}(\rho)$ with corresponding $\tau_{j}^{\prime \prime}$ s and if $\tau_{i(1)} \neq \tau_{j(1)}^{\prime}$, then $\beta \subset \tilde{\mathbf{g}}^{n-i(1)} \tau_{i(1)}$ and $\beta^{\prime} \subset \tilde{\mathbf{g}}^{n-j(1)} \tau_{j(1)}^{\prime}$ do not intersect a common stable leaf. In particular, $t \neq t^{\prime}$. A similar argument shows that all the elements of $Q_{n}(\rho)$ determine distinct covering translations.

Note that $Q_{n}(\rho)$ can also be defined recursively by

$$
Q_{n}(\rho)=\bigcup_{j=0}^{n-1} Q_{j} \circ P_{n-j}(\rho)
$$

it follows that

$$
\operatorname{Card}\left(Q_{n}\right)=\sum_{j=0}^{n-1}\left(\operatorname{Card}\left(Q_{j}\right) \times\left[d \lambda^{n-j-1}+1\right]\right) .
$$

Define $B_{n}=\sum_{j=0}^{n-1}\left(\operatorname{card} Q_{j}\right) d \lambda^{n-j-1}$. Then $U_{n}(\rho) \geq \operatorname{card}\left(Q_{n}\right) \geq B_{n}$ and

$$
\begin{aligned}
B_{n+1} & =\lambda B_{n}+d\left(\text { card } Q_{n}\right) \\
& \geq \lambda B_{n}+d B_{n} \\
& =(\lambda+d) B_{n} .
\end{aligned}
$$

Remark. The covering translations that occur in the admissible pairs counted in the proof of Lemma 3.5 are independent of the exact choice of $\rho$ and depend only on the fact that $\rho$ satisfies $(*)$.

The following corollary shows that the exponential growth rate of $N_{n}(g)$ is strictly greater than $\log \lambda$. Since there exists $\delta>0$ such that $N_{n}(g)$ is an $(n, \delta)$-separated set for all $n>0$, the topological entropy of $g$ is strictly greater than $\log \lambda$, the topological entropy of $f$.

COROllary 3.6. There exists $c>0$ such that $N_{n}(g)>c(\lambda+d)^{n}$.

Proof of Corollary 3.6. Choose an arc $\alpha$ that satisfies $(*)$. Let $(\rho, t)$ be one of the admissible pairs counted in the proof of Lemma 3.5 and let $\tilde{h}=t^{-1} \tilde{g}^{n}$; recall that $\rho \subset \tilde{g}^{n}(\alpha)$. It suffices to show that $\tilde{h}$ has fixed points and that there is a uniform bound (i.e. a bound independent of $n$ ) to the number of such $\tilde{h}$ that can be mutually conjugate.

There is a subarc $\alpha_{1}=\left(t^{-1} \rho\right)$ of $\tilde{h}(\alpha)$ that satisfies $(*)$. Similarly, for all $k \geq 1$ there are subarcs $\alpha_{k}$ of $\tilde{h}^{k}(\alpha)$ that satisfy (*). In particular, there exists a constant $K_{0}$ and $\tilde{x}_{k} \in \alpha$ such that $\tilde{D}\left(\tilde{h}^{k}\left(\tilde{x}_{k}\right), \tilde{x}_{k}\right)<K_{0}$ for all $k \geq 1$.

We claim that there exists $K_{1}>0$ such that $\tilde{D}\left(\tilde{h}^{i}\left(\tilde{x}_{k}\right), \tilde{x}_{k}\right)<K_{1}$ for $0 \leq i \leq k$ and all $k \geq 1$. Since $\tilde{D}_{s}\left(\tilde{h}^{i}\left(\tilde{x}_{k}\right), \tilde{x}_{k}\right)$ is uniformly bounded for all $i \geq 1$ and all $\tilde{x}_{k}$, it suffices to find $K_{2}>K_{0}$ so that if $\tilde{D}_{u}\left(\tilde{h}^{i}\left(\tilde{x}_{k}\right), \tilde{x}_{k}\right)>K_{2}$ then $\tilde{D}_{u}\left(\tilde{h}^{i+1}\left(\tilde{x}_{k}\right), \tilde{x}_{k}\right)>K_{2}$. The existence of $K_{2}$ follows from the fact that

$$
\begin{aligned}
\tilde{D}_{u}\left(\tilde{h}^{i+1}\left(\tilde{x}_{k}\right), \tilde{x}_{k}\right) & \geq \tilde{D}_{u}\left(\tilde{h}^{i+1}\left(\tilde{x}_{k}\right), \tilde{h}\left(\tilde{x}_{k}\right)\right)-\tilde{D}_{u}\left(\tilde{h}\left(\tilde{x}_{k}\right), \tilde{x}_{k}\right) \\
& \geq c_{2} \lambda^{n} \tilde{D}_{u}\left(\tilde{h}^{i}\left(\tilde{x}_{k}\right), \tilde{x}_{k}\right)-C_{1}
\end{aligned}
$$

where $C_{1}$ is greater than $\tilde{D}_{u}(\tilde{h}(\tilde{x}), \tilde{x})$ for all $\tilde{x} \in \alpha_{:}$and $\tilde{D}_{u}\left(\tilde{h}^{i}\left(\tilde{x}_{k}\right), \tilde{x}_{k}\right)>K$.

It follows immediately that the entire $\tilde{h}$-orbit of any accumulation point $\tilde{x}$ of the set $\left\{\tilde{h}^{[k / 2]}\left(\tilde{x}_{k}\right): k \geq 1\right\}$ is contained in the $K_{1}$-neighborhood of $\alpha$. The Brouwer Translation Theorem (see for example [F2]) implies that Fix $(\tilde{h})$ is non-empty. 
Moreover, for any fixed point $\tilde{y}$ of $\tilde{h}, \tilde{D}\left(\tilde{h}^{i}(\tilde{y}), \tilde{h}^{i}(\tilde{x})\right)$ is uniformly bounded for all $i \in \mathbb{Z}$. Thus $\tilde{D}(\tilde{y}, \tilde{x})<2 K$ and Fix $(\tilde{h})$ is contained in the $2 K+K_{1}$ neighborhood of $\alpha$. As there are only finitely many covering translations that do not move this neighborhood of $\alpha$ entirely off of itself, there is a uniform bound to the number of $\tilde{h}$ 's that are mutually conjugate.

\section{Extensions and counterexamples}

Fathi $[\mathbf{F 1}]$ generalized the results of $[\mathbf{H}]$ by replacing the hypothesis that $g \simeq f$ with the hypothesis that $g$ is a homeomorphism of a compact metric space $N$ for which there exists a non-nullhomotopic map $\alpha: N \rightarrow M$ satisfying $\alpha \circ g \simeq f \circ \alpha$. The conclusions are the same as in [H] except that $\pi: Y \rightarrow M$ is homotopic to $\alpha \mid Y$ rather than the inclusion of $Y$ into $M$. In this section we consider the extent to which Theorem 0.1 applies to this context. In particular, we assume throughout this section that $f, g$ and $\alpha$ are as above and that the entropy of $g$ equals that of $f$.

Fathi also showed that if $N$ is a closed surface and $g$ is pseudo-Anosov, then $Y=N$ and in fact $\pi: N \rightarrow M$ is a branched covering. (His proof uses the fact that a pseudo-Anosov homeomorphism supports a unique measure of maximal entropy and the support of this measure is the entire surface.) Theorem 0.1 therefore implies that if $N$ is a closed surface and $g$ is homotopic to a pseudo-Anosov homeomorphism, then $g$ is semi-conjugate to $f$. As Example 4.1 shows, the hypothesis that $g$ determines a pseudo-Anosov mapping class is essential.

If $\tilde{\alpha}: \tilde{N} \rightarrow \tilde{M}, \tilde{g}: \tilde{N} \rightarrow \tilde{N}$ and $\tilde{f}: \tilde{M} \rightarrow \tilde{M}$ are lifts to the universal covers such that $\tilde{\alpha} \circ \tilde{g}$ and $\tilde{f} \circ \tilde{\alpha}$ are equivariantly homotopic and if $\alpha$ is homotopic to a semiconjugating map $\pi$, then for all $\tilde{y} \in \tilde{N}$ there exists $\tilde{x} \in \tilde{M}$ and $C>0$ such that $\tilde{D}\left(\tilde{\alpha} \tilde{g}^{n}(\tilde{y}), \tilde{f}^{n}(\tilde{x})\right)<C$ for all $n \in \mathbb{Z}$. We will use this as a criterion for proving that for certain $g$ and $\alpha$, there are no maps $\pi \simeq \alpha$ that semi-conjugate $g$ to $f$.

Example 4.1. Fix a hyperbolic structure on $M$ and let $\Lambda^{s}$ and $\Lambda^{u}$ be the geodesic laminations determined by the stable and unstable foliations $F^{s}$ and $F^{u}$ determined by $f$. There is a standard construction (see for example [H-T] or [M]) of a homeomorphism $h: M \rightarrow M$ that preserves $\Lambda^{s}$ and $\Lambda^{u}$ and that is semi-conjugate to $f$ by a map $p \simeq$ identity. Choose a singularity $z$ of $F^{s}$ and $F^{u}$. We may assume that $p^{-1}(z) \subset$ Fix $(h)$ is a hexagon (or a $2 n$-gon for $n>3$ if $z$ is a more complicated singularity) and that for each point $v$ in a stable [respectively unstable] leaf initiating at $z, p^{-1}(v)$ is an arc of an unstable [respectively stable] leaf. See figure 4.1.
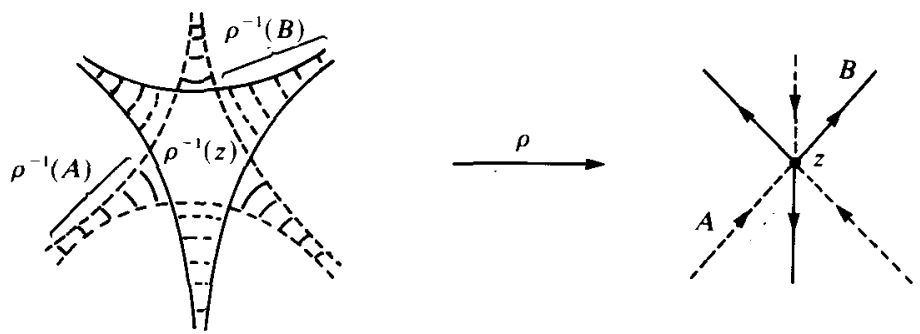

Figure 4.1 
Let $M^{\prime}$ be the surface obtained from $M$ by removing the interior of $p^{-1}(z)$ and let $N$ be the closed surface obtained by gluing together two copies $M_{1}^{\prime}$ and $M_{2}^{\prime}$ where $\partial M_{1}^{\prime}$ and $\partial M_{2}^{\prime}$ are identified by a homeomorphism that carries $\mathrm{cl}\left(p_{1}^{-1}(A)\right) \cap$ $\partial M_{1}^{\prime}$ to $\operatorname{cl}\left(p_{2}^{-1}(B)\right) \cap \partial M_{2}^{\prime}$ where $A$ and $B$ are non-adjacent stable and unstable leaf segments initiating at $z$. See figure 4.2 where $C$ is the image in $N$ of $\operatorname{cl}\left(p_{1}^{-1}(A)\right) \cap \partial M_{1}^{\prime}$.

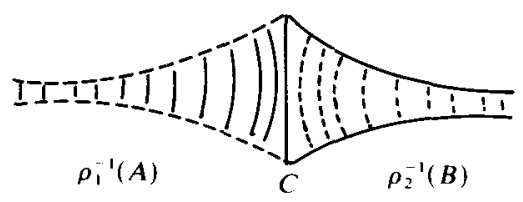

FIGURE 4.2

Then $h_{1}: M_{1}^{\prime} \rightarrow M_{1}^{\prime}$ and $h_{2}: M_{2}^{\prime} \rightarrow M_{2}^{\prime}$ fit together to define a homeomorphism $H: N \rightarrow N$ and $p_{1}: M_{1}^{\prime} \rightarrow M$ and $p_{2}: M_{2}^{\prime} \rightarrow M$ fit together to define a map $\alpha: N \rightarrow M$ such that $f \circ \alpha=\alpha \circ H$. There is a foliation (see figure 4.2) of $U=p_{1}^{-1}(A) \cup p_{2}^{-1}(B) \cup$ $C$ where the leaves are given by

$$
\left\{p_{1}^{-1}(v): v \in A\right\} \cup\left\{p_{2}^{-1}(w): w \in B\right\} \cup\{C\} .
$$

Let $X$ be a vector field on $N$ supported on a neighborhood of $C$ in $U$ such that $X$ is transverse to the foliation and points in the direction of $p_{2}^{-1}(B)$. Define $g=\phi \circ H$ where $\phi$ is the time one map of the flow generated by $X$.

Since $g$ and $H$ agree on their non-wandering sets, the entropy of $g$ equals that of $f$. On the other hand, there are lifts

$$
\tilde{z} \in \tilde{M}, \tilde{f}: \tilde{M} \rightarrow \tilde{M}, \tilde{C} \subset \tilde{N}, \tilde{H}: \tilde{N} \rightarrow \tilde{N}, \tilde{g}: \tilde{N} \rightarrow \tilde{N} \text { and } \tilde{\alpha}: \tilde{N} \rightarrow \tilde{M}
$$

such that $\tilde{f}$ fixes $\tilde{z}, \tilde{H}$ fixes $\tilde{C}, \tilde{g}$ is equivariantly homotopic to $\tilde{H}$ and $\tilde{\alpha} \circ \tilde{H}=$ $\tilde{f} \circ \tilde{\alpha}$. Let $\tilde{A}, \tilde{B} \subset \tilde{M}$ be the lifts of $A$ and $B$ that initiate at $\tilde{z}$. Then for each $\tilde{y} \in \tilde{C}$ there exists $N>0$ such that $\left\{\alpha \tilde{g}^{n}(\tilde{y}): n>N\right\}$ agrees with an $\tilde{f}$-orbit in $\tilde{B}$ and $\left\{\alpha \tilde{g}^{-n}(\tilde{y}): n>N\right\}$ agrees with an $\tilde{f}$-orbit in $\tilde{A}$. In particular,

$$
\sup \left\{\tilde{D}\left(\tilde{f}^{n}(\tilde{x}), \alpha \tilde{g}^{n}(\tilde{y})\right): n \in \mathbb{Z}\right\}=\infty \text { for all } \tilde{x} \in \tilde{M}
$$

and $g$ is not semi-conjugate to $f$ by a map that is homotopic to $\alpha$.

Our final example shows that Theorem 0.1 may fail in the general context even if $\alpha$ is a homotopy equivalence.

Example 4.2. Let $N=M \times[0,1]$ and let $G_{t}$ be an isotopy of $f$ to itself such that $G_{1 / 2}$ contains a fixed point $P$ that is not Nielsen equivalent to any fixed point of $f$. Choose a function $u: N \rightarrow[0,1]$ such that $u^{-1}(0)=M \times\{0,1\} \cup P \times\left\{\frac{1}{2}\right\}$ and let $H(y, t)$ be the time one map of the flow generated by the vector field $u \cdot \partial / \partial t$. Then $g(y, t)=H \circ\left(G_{t}(y), t\right)$ is a homeomorphism of $N$ with non-wandering set $M \times\{0,1\} \cup P \times\left\{\frac{1}{2}\right\}$ and therefore with entropy equal to that of $f$. Define $\alpha$ equal to the projection of $N$ onto $M$. Choose lifts $\tilde{\alpha}, \tilde{f}, \tilde{g}$ and $\tilde{P}$ such that $\tilde{\alpha} \circ \tilde{g}$ and $\tilde{f} \circ \tilde{\alpha}$ are equivariantly homotopic and such that $\tilde{\boldsymbol{g}}$ fixes $\tilde{\boldsymbol{P}} \times\left\{\frac{1}{2}\right\}$. The Brouwer translation theorem and the choice of $\tilde{P}$ imply that no orbit of $\tilde{f}$ is bounded. Since 
$\tilde{\alpha} \tilde{g}^{n}\left(\tilde{P} \times\left\{\frac{1}{2}\right\}\right)=\tilde{P}$ for all $n \in \mathbb{Z}, g$ is not semi-conjugate to $f$ by any map that is homotopic to $\alpha$.

\section{REFERENCES}

[F1] A. Fathi. Homotopical stability of pseudo-Anosov maps. Preprint.

[F2] A. Fathi. An orbit closing proof of Brouwer's lemma on translation arcs. Preprint.

[F-L-P] A. Fathi, F. Laudenbach \& V. Poenaru. Travaux de Thurston sur les surfaces. Asterisque 66-67 (1979).

[Fr] J. Franks. Anosov diffeomorphisms. Proceedings of the Symposium in Pure Mathematics 14 (1968) 61-94.

[H] M. Handel. Global shadowing of pseudo-Anosov diffeomorphisms. Ergod. Th. \& Dynam. Sys. 5 (1985), 373-377.

[H-T] M. Handel \& W. Thurston. New proofs of some results of Nielsen. Adv. Math. 56 (1985), 173-191.

[M] R. T. Miller. Nielsen's viewpoint on geodesic laminations. Adv. in Math. 45 (1982), 189-212.

[T] W. Thurston. On the geometry and dynamics of diffeomorphisms of surfaces. Preprint. 